\title{
Energy of commuting graph of finite AC-groups
}

\author{
R. Sharafdini \\ Persian Gulf University, Iran \\ Rajat Kanti Nath \\ Tezpur University, India \\ and \\ Rezvan Darbandi \\ Amirkabir University of Technology, Iran \\ Received : July 2020. Accepted : December 2021
}

\begin{abstract}
Let $\Gamma$ be a graph with the adjacency matrix $A$. The energy of $\Gamma$ is the sum of the absolute values of the eigenvalues of $A$. In this article we compute the energies of the commuting graphs of some finite groups and discuss some consequences regarding hyperenergetic and borderenergetic graphs.
\end{abstract}

2000 Mathematics Subject Classification: 05C12, 05C25, 20D60, $05 A 15$.

Keywords: Commuting graph, non-commuting graph, AC-group, Eigenvalue, Energy. 


\section{Introduction}

The commuting graph of a non-Abelian group $G$ with center $Z(G)$ is an undirected graph with vertex set $G \backslash Z(G)$, and two distinct vertices $a$ and $b$ are adjacent whenever $a b=b a$. We write $\Gamma_{G}$ to denote this graph. Various properties of $\Gamma_{G}$ have been studied in [3],[4],[15],[16],[17] and [20]. It is noted in [19] that the complement of $\Gamma_{G}$, known as non-commuting graph of $G$ (see [1]), was first considered by Erdös in 1975. Let $g$ be any element of a group $G$. Then the subgroup $C_{G}(g)=\{a \in G \mid a g=g a\}$ is called centralizer of $g$. If $C_{G}(g)$ is Abelian for every $g \in G \backslash Z(G)$ then the group $G$ is called an AC-group. If $\operatorname{Cent}(G)=\left\{C_{G}(g) \mid g \in G\right\}$ and $|\operatorname{Cent}(G)|=n$ then $G$ is called an $n$-centralizer group (see [5] and [2]).

Let $\Gamma$ be a graph with the adjacency matrix $A$. Let $\lambda_{1}, \lambda_{2}, \ldots, \lambda_{t}$ be the distinct eigenvalues of $A$ with corresponding mutiplicites $m_{1}, m_{2}, \ldots, m_{t}$. The spectrum of $\Gamma$ is defined as

$$
\operatorname{Spec}(\Gamma)=\left\{\lambda_{1}^{m_{1}}, \lambda_{2}^{m_{2}}, \ldots, \lambda_{t}^{m_{t}}\right\} .
$$

The energy of $\Gamma$ (see [12] and [14]) is given by

$$
\mathcal{E}(\Gamma)=\sum_{i=1}^{t} m_{i}\left|\lambda_{i}\right|
$$

It may be mentioned here that $\mathcal{E}\left(K_{n}\right)=2 n-2$, where $K_{n}$ is the complete graph on $n$ vertices. Spectral aspects of $\Gamma_{G}$ are considered in [6], [7], [8], [9], [10] and [18] recently for various families of finite groups. In this paper we compute energy of $\Gamma_{G}$ for several classes of finite AC-groups and discuss some consequences regarding hyperenergetic and borderenergetic graphs. Throughout the paper same notations are used to denote various families of groups as in [6] and [7].

\section{Results and consequences}

In this section, we compute the energy of $\Gamma_{G}$ for some particular families of finite non-Abelian groups and derive some consequences. Using (1.1) and the spectrum related results available in [6] and [7] we get the following results. 
Theorem 1. We have

(i) $\mathcal{E}\left(\Gamma_{M_{2 m n}}\right)= \begin{cases}4 m n-2 m-2 n-2, & \text { if } m \text { is odd } \\ 4 m n-4 n-m-2, & \text { if } m \text { is even. }\end{cases}$

(ii) $\mathcal{E}\left(\Gamma_{D_{2 m}}\right)= \begin{cases}2 m-4, & \text { if } m \text { is odd } \\ 3 m-6, & \text { if } m \text { is even. }\end{cases}$

(iii) $\mathcal{E}\left(\Gamma_{Q_{4 m}}\right)=6 m-3$.

(iv) $\mathcal{E}\left(\Gamma_{U_{6 n}}\right)=10 n-8$.

Theorem 2. Let $G$ be a finite group. Then we have the following.

(i) If $G=Q D_{2^{n}}$ then $\mathcal{E}\left(\Gamma_{G}\right)=2^{n}+2^{n-1}-6$.

(ii) If $G=P S L\left(2,2^{k}\right)$ then $\mathcal{E}\left(\Gamma_{G}\right)=2^{3 k+1}-2^{2 k+1}-2.2^{k+1}-4$.

(iii) If $G=G L(2, q)$ then $\mathcal{E}\left(\Gamma_{G}\right)=2 q^{4}-2 q^{3}-4 q^{2}-2 q$.

(iv) If $\frac{G}{Z(G)} \cong S z(2)$ then $\mathcal{E}\left(\Gamma_{G}\right)=38|Z(G)|-12$.

(v) If $G=A(n, \vartheta)$ then $\mathcal{E}\left(\Gamma_{G}\right)=2\left(2^{n}-1\right)^{2}$.

(vi) If $G=A(n, p)$ then

$$
\mathcal{E}\left(\Gamma_{G}\right)=\left(p^{3 n}-2 p^{n}-1\right)+\left(p^{n}+1\right)\left(p^{2 n}-p^{n}-1\right)=2 p^{3 n}-4 p^{n}-2 .
$$

(vii) If $G$ is non-Abelian and $|G|$ is product of two primes $p, q$ such that $p \mid(q-1)$ then $\mathcal{E}\left(\Gamma_{G}\right)=2 q(p-1)-3$.

In the following theorem we compute energy of commuting graphs of the groups $G$ such that $\frac{G}{Z(G)}$ is isomorphic to $\mathbf{Z}_{p} \times \mathbf{Z}_{p}$ and $D_{2 m}$.

Theorem 3. Let $G$ be a finite group.

(i) If $p$ is a prime and $\frac{G}{Z(G)} \cong \mathbf{Z}_{p} \times \mathbf{Z}_{p}$, then $\mathcal{E}\left(\Gamma_{G}\right)=2\left(\left(p^{2}-1\right)|Z(G)|-\right.$ $p-1)$.

(ii) If $m(\geq 2)$ is a natural number and $\frac{G}{Z(G)} \cong D_{2 m}$, then $\mathcal{E}\left(\Gamma_{G}\right)=$ $2((2 m-1)|Z(G)|-m-1)$.

As applications of Theorem 3 we also have the following two corollaries. 
Corollary 1. Let $p$ be a prime.

(i) If $G$ is a non-Abelian group and $|G|=p^{3}$, then $\mathcal{E}\left(\Gamma_{G}\right)=2\left(p^{3}-2 p-1\right)$.

(ii) If $G$ is a 4-centralizer finite group, then $\mathcal{E}\left(\Gamma_{G}\right)=6(|Z(G)|-1)$.

(iii) If $G$ is a $(p+2)$-centralizer finite $p$-group, then

$$
\mathcal{E}\left(\Gamma_{G}\right)=2\left(\left(p^{2}-1\right)|Z(G)|-p-1\right) .
$$

(iv) If $G$ is a 5 -centralizer finite group, then

$$
\mathcal{E}\left(\Gamma_{G}\right) \in\{8(2|Z(G)|-1), 10|Z(G)|-8\} .
$$

Proof. (i) In this case $|Z(G)|=p$ and $\frac{G}{Z(G)} \cong \mathbf{Z}_{p} \times \mathbf{Z}_{p}$. Hence the result follows from Theorem 3(i).

(ii) The result follows from Theorem $3(\mathrm{i})$, noting that $\frac{G}{Z(G)} \cong \mathbf{Z}_{2} \times \mathbf{Z}_{2}$ (see [5, Theorem 2]).

(iii) We have $\frac{G}{Z(G)} \cong \mathbf{Z}_{p} \times \mathbf{Z}_{p}$ (see [2, Lemma 2.7]). Hence the result follows from Theorem 3(i).

(iv) By $\left[5\right.$, Theorem 4] we have $\frac{G}{Z(G)} \cong \mathbf{Z}_{3} \times \mathbf{Z}_{3}$ or $D_{6}$. If $\frac{G}{Z(G)} \cong \mathbf{Z}_{3} \times \mathbf{Z}_{3}$, then by Theorem $3(\mathrm{i})$ we have $\mathcal{E}\left(\Gamma_{G}\right)=8(2|Z(G)|-1)$. In the case that $\frac{G}{Z(G)} \cong D_{6}$, by Theorem 3(ii) we have $\mathcal{E}\left(\Gamma_{G}\right)=10|Z(G)|-8$.

Corollary 2. If a group $G$ is isomorphic to any of the following groups

(i) $\mathbf{Z}_{2} \times D_{8}$

(ii) $\mathbf{Z}_{2} \times Q_{8}$

(iii) $G_{16}=\left\langle x, y \mid x^{8}=y^{2}=1, y x y=x^{5}\right\rangle$

(iv) $H_{16}=\left\langle x, y \mid x^{4}=y^{4}=1, y x y^{-1}=x^{-1}\right\rangle$

(v) $D_{8} * \mathbf{Z}_{4}=\left\langle x, y, c \mid x^{4}=y^{2}=c^{2}=1, x y=y x, x c=c x, y c=x^{2} c y\right\rangle$

(vi) $S G(16,3)=\left\langle x, y \mid x^{4}=y^{4}=1, x y=y^{-1} x^{-1}, x y^{-1}=y x^{-1}\right\rangle$,

then $\mathcal{E}\left(\Gamma_{G}\right)=18$. 
Proof. We have $|G|=16$ and $|Z(G)|=4$. Therefore, $\frac{G}{Z(G)} \cong \mathbf{Z}_{2} \times \mathbf{Z}_{2}$. Hence, the result follows from Theorem 3(i).

Note that all the groups considered in this section so far are non-Abelian AC-groups. We conclude this section by computing the energy of commuting graph of a finite non-Abelian AC-group in general. The following lemma is useful.

Lemma 1. Let $C_{1}, \ldots, C_{n}$ be the centralizers of non-central elements of a finite non-Abelian group $G$. Then

$$
\sum_{i=1}^{n}\left|C_{i}\right|=|G|+(n-1)|Z(G)|
$$

Proof. By [7, Lemma 2.1] we have $\Gamma_{G}=\bigsqcup_{i=1}^{n} K_{\left|C_{i}\right|-|Z(G)|}$. Therefore, the number of vertices in $\Gamma_{G}$ is given by

$$
\sum_{i=1}^{n}\left|C_{i}\right|-n|Z(G)|=|G|-|Z(G)|
$$

Hence the lemma follows.

Theorem 4. Let $G$ be a finite non-Abelian AC-group with $n$ distinct centralizers of non-central elements. Then

$$
\mathcal{E}\left(\Gamma_{G}\right)=2(|G|-|Z(G)|-n) .
$$

Proof. Let $C_{1}, \ldots, C_{n}$ be the distinct centralizers of non-central elements of $G$. Then by [7, Theorem 2.1] we have

$$
\mathcal{E}\left(\Gamma_{G}\right)=2 \sum_{i=1}^{n}\left|C_{i}\right|-2 n(|Z(G)|+1) .
$$

Hence, the result follows using Lemma 1.

Corollary 3. Let $A$ be any finite Abelian group and $G$ a finite non-Abelian $A C$-group. Then the energy of the commuting graph of $G \times A$ is given by

$$
\mathcal{E}\left(\Gamma_{G \times A}\right)=2(|G||A|-|Z(G)||A|-n),
$$

where $n$ is as given in Theorem 4. 
A finite graph $\Gamma$ is called hyperenergetic and borderenergetic if $\mathcal{E}(\Gamma)>$ $\mathcal{E}\left(K_{|v(\Gamma)|}\right)$ and $\mathcal{E}(\Gamma)=\mathcal{E}\left(K_{|v(\Gamma)|}\right)$ respectively, where $v(\Gamma)$ is the set of vertices of $\Gamma$. The study of hyperenergetic graph was initiated by Walikar et al. [21] and Gutman [13] in 1999. However, the concept of borderenergetic graph was introduced by Gong et al. [11] in the year 2015. Since then, it becomes an interesting question to determine whether a given graph is hyperenergetic or borderenergetic. As consequences of above results, we now show that the commuting graphs of the groups considered above are neither hyperenergetic nor borderenergetic. We begin with the following result.

Proposition 1. If $G=M_{2 m n}, D_{2 m}, Q_{4 m}$ and $U_{6 n}$ then $\Gamma_{G}$ is neither hyperenergetic nor borderenergetic.

Proof. If $G=M_{2 m n}$ then $\left|v\left(\Gamma_{G}\right)\right|= \begin{cases}2 m n-n, & \text { if } n \text { is odd } \\ 2 m n-2 n, & \text { if } n \text { is even. }\end{cases}$

Therefore, $\mathcal{E}\left(K_{\left|v\left(\Gamma_{G}\right)\right|}\right)= \begin{cases}4 m n-2 n-2, & \text { if } n \text { is odd } \\ 4 m n-4 n-2, & \text { if } n \text { is even. }\end{cases}$

We have

$4 m n-2 m-2 n-2<4 m n-2 n-2$ and $4 m n-4 n-m-2<4 m n-4 n-2$.

Hence, by Theorem 1(i), $\Gamma_{M_{2 m n}}$ is neither hyperenergetic nor borderenergetic.

If $G=D_{2 m}$ then $\left|v\left(\Gamma_{G}\right)\right|= \begin{cases}2 m-1, & \text { if } m \text { is odd } \\ 2 m-2, & \text { if } m \text { is even. }\end{cases}$

Therefore, $\mathcal{E}\left(K_{\left|v\left(\Gamma_{G}\right)\right|}\right)= \begin{cases}4 m-4, & \text { if } m \text { is odd } \\ 4 m-6, & \text { if } m \text { is even. }\end{cases}$

We have

$$
2 m-4<4 m-4 \text { and } 3 m-6<4 m-6 \text {. }
$$

Hence, by Theorem 1(ii), $\Gamma_{D_{2 m}}$ is neither hyperenergetic nor borderenergetic.

If $G=Q_{4 m}$ then $\left|v\left(\Gamma_{G}\right)\right|=4 m-2$. Therefore, $\mathcal{E}\left(K_{\left|v\left(\Gamma_{G}\right)\right|}\right)=8 m-6$. We have

$$
6 m-3<8 m-6 \text {. }
$$

Hence, by Theorem 1(iii), $\Gamma_{Q_{2 m}}$ is neither hyperenergetic nor borderenergetic. 
If $G=U_{6 n}$ then $\left|v\left(\Gamma_{G}\right)\right|=5 n$. Therefore, $\mathcal{E}\left(K_{\left|v\left(\Gamma_{G}\right)\right|}\right)=10 n-2$. We have

$$
10 n-8<10 n-2 \text {. }
$$

Hence, by Theorem 1(iv), $\Gamma_{U_{6 n}}$ is neither hyperenergetic nor borderenergetic.

Proposition 2. If $G=Q D_{2^{n}}, P S L\left(2,2^{k}\right), G L(2, q), A(n, \vartheta)$ and $A(n, p)$ then $\Gamma_{G}$ is neither hyperenergetic nor borderenergetic.

Proof. If $G=Q D_{2^{n}}$ then $\left|v\left(\Gamma_{G}\right)\right|=2^{n}-2$. Therefore, $\mathcal{E}\left(K_{\left|v\left(\Gamma_{G}\right)\right|}\right)=$ $2.2^{n}-6$. We have

$$
2^{n}+2^{n-1}-6<2.2^{n}-6 .
$$

Hence, by Theorem 2(i), $\Gamma_{Q D_{2} n}$ is neither hyperenergetic nor borderenergetic.

If $G=\operatorname{PSL}\left(2,2^{k}\right)$ then $\left|v\left(\Gamma_{G}\right)\right|=2^{k}\left(2^{2 k}-1\right)$. Therefore, $\mathcal{E}\left(K_{\left|v\left(\Gamma_{G}\right)\right|}\right)=$ $2^{3 k+1}-2^{k+1}-2$. We have

$$
2^{3 k+1}-2^{2 k+1}-2.2^{k+1}-4<2^{3 k+1}-2^{k+1}-2 .
$$

Hence, by Theorem 2(ii), $\Gamma_{P S L\left(2,2^{k}\right)}$ is neither hyperenergetic nor borderenergetic.

If $G=G L(2, q)$ then $\left|v\left(\Gamma_{G}\right)\right|=\left(q^{2}-1\right)\left(q^{2}-q\right)-q-1=q^{4}-q^{3}-q^{2}-1$. Therefore, $\mathcal{E}\left(K_{\left|v\left(\Gamma_{G}\right)\right|}\right)=2 q^{4}-2 q^{3}-2 q^{2}-4$. We have

$$
2 q^{4}-2 q^{3}-4 q^{2}-2 q<2 q^{4}-2 q^{3}-2 q^{2}-4 .
$$

Hence, by Theorem 2(iii), $\Gamma_{G L(2, q)}$ is neither hyperenergetic nor borderenergetic.

If $G=A(n, \vartheta)$ then $\left|v\left(\Gamma_{G}\right)\right|=2^{n}\left(2^{n}-1\right)$. Therefore, $\mathcal{E}\left(K_{\left|v\left(\Gamma_{G}\right)\right|}\right)=$ $2.2^{2 n}-2.2^{n}-2$. We have

$$
2.2^{2 n}-4.2^{n}+2<2.2^{2 n}-2.2^{n}-2 .
$$

Hence, by Theorem $2(\mathrm{v}), \Gamma_{A(n, \vartheta)}$ is neither hyperenergetic nor borderenergetic.

If $G=A(n, p)$ then $\left|v\left(\Gamma_{G}\right)\right|=\left(p^{2 n}-p^{n}\right)\left(p^{n}+1\right)$. Therefore, $\mathcal{E}\left(K_{\left|v\left(\Gamma_{G}\right)\right|}\right)$ $=2 \cdot p^{3 n}-2 \cdot p^{n}-2$. We have

$$
2 \cdot p^{3 n}-4 \cdot p^{n}-2<2 \cdot p^{3 n}-2 \cdot p^{n}-2 .
$$

Hence, by Theorem 2(vi), $\Gamma_{A(n, p)}$ is neither hyperenergetic nor borderenergetic. 
Proposition 3. If $G$ is a non-Abelian group and $|G|$ is product of two primes $p, q$ such that $p \mid(q-1)$ then $\Gamma_{G}$ is neither hyperenergetic nor borderenergetic.

Proof. We have $\left|v\left(\Gamma_{G}\right)\right|=p q-1$. Therefore, $\mathcal{E}\left(K_{\left|v\left(\Gamma_{G}\right)\right|}\right)=2 p q-4$. Also

$$
2 p q-2 q-3<2 p q-4
$$

Hence, by Theorem 2(vii), the result follows.

Proposition 4. $\Gamma_{G}$ is neither hyperenergetic nor borderenergetic if $\frac{G}{Z(G)} \cong$ $S z(2), \mathbf{Z}_{p} \times \mathbf{Z}_{p}$ and $D_{2 m}$.

Proof. If $\frac{G}{Z(G)} \cong S z(2)$ then $\left|v\left(\Gamma_{G}\right)\right|=19|Z(G)|$. Therefore, $\mathcal{E}\left(K_{\left|v\left(\Gamma_{G}\right)\right|}\right)=$ $38|Z(G)|-2$. We have

$$
38|Z(G)|-12<38|Z(G)|-2 .
$$

Hence, by Theorem 2(iv), $\Gamma_{G}$ is neither hyperenergetic nor borderenergetic.

If $\frac{G}{Z(G)} \cong \mathbf{Z}_{p} \times \mathbf{Z}_{p}$ then $\left|v\left(\Gamma_{G}\right)\right|=|Z(G)| p^{2}-|Z(G)|$. Therefore, $\mathcal{E}\left(K_{\left|v\left(\Gamma_{G}\right)\right|}\right)=2|Z(G)| p^{2}-2|Z(G)|-2$. We have

$$
2|Z(G)| p^{2}-2|Z(G)|-p-1<2|Z(G)| p^{2}-2|Z(G)|-2 .
$$

Hence, by Theorem 3(i), $\Gamma_{G}$ is neither hyperenergetic nor borderenergetic.

If $\frac{G}{Z(G)} \cong D_{2 m}$ then $\left|v\left(\Gamma_{G}\right)\right|=(2 m-1)|Z(G)|$. Therefore, $\mathcal{E}\left(K_{\left|v\left(\Gamma_{G}\right)\right|}\right)=$ $2(2 m-1)|Z(G)|-2$. We have

$$
2(2 m-1)|Z(G)|-2 m-2<2(2 m-1)|Z(G)|-2 .
$$

Hence, by Theorem 3(ii), $\Gamma_{G}$ is neither hyperenergetic nor borderenergetic.

Corollary 4. Let $G$ be a finite non-Abelian group and $p$ be any prime. Then $\Gamma_{G}$ is neither hyperenergetic nor borderenergetic if

1. $G$ is of order $p^{3}$.

2. $G$ is a 4-centralizer group.

3. $G$ is a 5 -centralizer group.

4. $G$ is a $(p+2)$-centralizer $p$-group. 
Proof. The result follows from Proposition 4 since $\frac{G}{Z(G)}$ is isomorphic to either $\mathbf{Z}_{p} \times \mathbf{Z}_{p}$ or $D_{6}$.

Corollary 5. $\Gamma_{G}$ is neither hyperenergetic nor borderenergetic if $G$ is given by $\mathbf{Z}_{2} \times D_{8}, \mathbf{Z}_{2} \times Q_{8}, G_{16}, H_{16}, D_{8} * \mathbf{Z}_{4}$ and $S G(16,3)$.

Proof. The result follows from Proposition 4 since $\frac{G}{Z(G)}$ is isomorphic to $\mathbf{Z}_{2} \times \mathbf{Z}_{2}$.

In general, we have the following theorem.

Theorem 5. If $G$ is a finite non-Abelian AC-group then $\Gamma_{G}$ is neither hyperenergetic nor borderenergetic.

Proof. We have $\left|v\left(\Gamma_{G}\right)\right|=|G|-|Z(G)|$. Therefore, $\mathcal{E}\left(K_{\left|v\left(\Gamma_{G}\right)\right|}\right)=$ $2|G|-2|Z(G)|-2$. We have

$$
2|G|-2|Z(G)|-2 n<2|G|-2|Z(G)|-2 .
$$

Hence, by Theorem $4, \Gamma_{G}$ is neither hyperenergetic nor borderenergetic.

We conclude this paper with a question given below.

Question 1. Is it true that the commuting graph of any finite non-Abelian group is neither hyperenergetic nor borderenergetic?

\section{Acknowledgement}

The authors express their gratitude to the referee for his/her valuable comments.

\section{References}

[1] A. Abdollahi, S. Akbari, and H. R. Maimani, "Non-commuting graph of a group", Journal of algebra, vol. 298, no. 2, pp. 468-492, 2006. doi: 10.1016/j.jalgebra.2006.02.015

[2] A. R. Ashrafi, "On finite groups with a given number of centralizers”, Algebra colloquium, vol. 7, no. 2, pp. 139-146, 2000. doi: 10.1007/ s10011-000-0139-5

[3] S. Akbari, A. Mohammadian, H. Radjavi, and P. Raja, "On the diameters of commuting graphs", Linear algebra and its applications, vol. 418, no. 1, pp. 161-176, 2006. doi: 10.1016/j.laa.2006.01.029 
[4] C. Bates, D. Bundy, S. Hart, and P. Rowley, "A note on commuting graphs for symmetric groups", The electronic journal of combinatorics, vol. 16, no. 1, pp. 1-13, 2009. doi: 10.37236/ 95

[5] S. M. Belcastro and G. J. Sherman, "Counting centralizers in finite groups", Mathematics magazine, vol. 67, no. 5, pp. 366-374, 1994. doi: 10.1080/ 0025570x.1994.11996252

[6] J. Dutta and R. K. Nath, "Spectrum of commuting graphs of some classes of finite groups", Matematika, vol. 33, no. 1, p. 87, 2017. doi: 10.11113/ matematika.v33.n1.812

[7] J. Dutta and R. K. Nath, "Finite groups whose commuting graphs are integral", Matematicki vesnik, vol. 69, no. 3, pp. 226-230, 2017. [On line]. Available: https:/ / bit.ly/ 3fSj949

[8] J. Dutta and R. K. Nath, "Laplacian and signless Laplacian spectrum of commuting graphs of finite groups", Khayyam journal of mathematics, vol. 4, no. 1, pp. 77-87, 2018. doi: 10.22034/ KJM.2018.57490

[9] P. Dutta, J. Dutta, and R. K. Nath, "Laplacian spectrum of non-commuting graphs of finite groups", Indian journal of pure and applied mathematics, vol. 49, no. 2, pp. 205-216, 2018. doi: 10.1007/ s13226-018-0263-x

[10] P. Dutta and R. K. Nath, "On Laplacian energy of non-commuting graphs of finite groups", Journal of linear and topological algebra, vol. 7, no. 2, pp. 121-132, 2018. [On line]. Available: https:/ / bit.ly/ 3FJLvZb

[11] S. C. Gong, X. Li, G. H. Xu, I. Gutman and B. Furtula, "Borderenergetic graphs", MATCH communications in mathematical and in computer chemistry, vol. 74, no. 2, pp. 321-332, 2015. [On line]. Available: https:/ / bit.ly/ 33ziuT1

[12] I. Gutman, "The energy of a graph", Berichte der Mathematischstatistischen Sektion im Forschungszentrum Graz, vol. 103, pp. 1-22, 1978.

[13] I. Gutman, "Hyperenergetic molecular graphs", Journal of the serbian chemical society, vol. 64, pp. 199-205, 1999.

[14] X. Li, Y. Shi, I. Gutman, Graph energy, New York: Springer, 2012.

[15] A. Iranmanesh and A. Jafarzadeh, "Characterization of finite groups by their commuting graph", Acta mathematica academiae paedagogiace nyíregyháziensis, vol. 23, no. 1, pp. 7-13, 2007. [On line]. Available: https:/ / bit.ly/ 33DpONi

[16] A. Iranmanesh and A. Jafarzadeh, "On the commuting graph associated with the symmetric and alternating groups", Journal of algebra and its applications, vol. 7, no. 1, pp. 129-146, 2008. doi: 10.1142/s02194 98808002710 
[17] G. L. Morgan and C. W. Parker, "The diameter of the commuting graph of a finite group with Trivial Centre", Journal of algebra, vol. 393, pp. 41-59, 2013. doi: 10.1016/j.jalgebra.2013.06.031

[18] R. K. Nath, "Various spectra of commuting graphs of N-centralizer finite groups", International Journal of Engineering Science and Technology, vol. 10, no. 2S, pp. 170-172, 2018. doi: 10.21817/ ijest/2018/v10i2s/ $181002 \mathrm{~s} 030$

[19] B. H. Neumann, "A problem of Paul Erdös on groups", Journal of the Australian mathematical society, vol. 21, no. 4, pp. 467-472, 1976. doi: 10.1017/ s1446788700019303

[20] C. Parker, "The commuting graph of a soluble group", Bulletin of the London mathematical society, vol. 45, no. 4, pp. 839-848, 2013. doi: $10.1112 /$ blms/ bdt005

[21] H. B. Walikar, H. S. Ramane and P. R. Hampiholi, "On the energy of agraph", in Graph Connections, R. Balakrishnan, H. M. Mulder and A. Vijayakumar, Eds. New Delhi: Allied, 1999, pp. 120-123.

\author{
Reza Sharafdini \\ Department of Mathematics, \\ Faculty of Science, \\ Persian Gulf University, \\ Bushehr 75169-13817, \\ Iran, \\ e-mail: sharafdini@pgu.ac.ir

\section{Rajat Kanti Nath} \\ Department of Mathematical Science, \\ Tezpur University, \\ Napaam-784028, \\ Sonitpur, Assam, \\ India \\ e-mail: rajatkantinath@yahoo.com \\ Corresponding author \\ and

\section{Rezvan Darbandi} \\ Department of Mathematics and Computer Science, \\ Amirkabir University of Technology, \\ Tehran, \\ Iran, \\ e-mail: rez1.darnadi@aut.ac.ir
}

